\title{
Morphological variation of mutant sunflowers (Helianthus annuus) induced by space flight and their genetic background detection by SSR primers
}

\author{
J. Yang ${ }^{1,2,3}$, S. Shen ${ }^{2}$, T. Zhang ${ }^{2}$, G.D. Chen' ${ }^{2}$ H. Liu' ${ }^{2}$ X.B. Ma ${ }^{2}$, \\ W.Y. Chen ${ }^{2}$ and Z.S. Peng' ${ }^{2,3}$ \\ ${ }^{1}$ Institute of Rare Animals and Plants, China West Normal University, \\ Nanchong, Sichuan, China \\ ${ }^{2}$ College of Life Science, China West Normal University, Nanchong, \\ Sichuan, China \\ ${ }^{3}$ Key Laboratory of Southwest China Wildlife Resources Conservation, \\ Ministry of Education, China West Normal University, Nanchong, \\ Sichuan, China \\ Corresponding author: J. Yang \\ E-mail: yangjunlz@tom.com
}

Genet. Mol. Res. 11 (3): 3379-3388 (2012)

Received June 28, 2011

Accepted October 25, 2012

Published September 25, 2012

DOI http://dx.doi.org/10.4238/2012.September.25.6

\begin{abstract}
After sunflower seeds were exposed to space conditions, various mutant plants were screened from the descendent plants. The morphological characters of plants changed in flower color from golden to yellow, light yellow, or even to yellowish green. The ligulate petals of the unisexual floret broadened, or became thin, while the short tubular petals of bisexual floret elongated to some extent, or even turned into semi-ligulate petals or ligulate petals, making the phenotype of the whole inflorescence like a chrysanthemum. The shape and thickness of leaves varied in some of these plants. Absolute sterile plants in mutant plants were found to possess neither normal bisexual florets nor unisexual florets, but the "pseudo-floret" only consisted of pieces of shield-like bracts on protuberant floral disc. Thirty-five pairs of simple sequence of repeat primers were used to detect the genetic variation of
\end{abstract}


the plants, and the results showed that only a variation was tested in the mutant plants from 4 primers. The different PCR products obtained were extracted for sequencing and alignment analysis, and the aligned results showed that the DNA sequence changed by deletion, insertion and replacement that occurred at some sites. The results proved the high mutagenic efficacy of space flight, and ways of DNA transformation due to space conditions.

Key words: Sunflower; Space flight; Morphological character; Simple sequence repeat; Alignment analysis

\section{INTRODUCTION}

The sunflower (Helianthus annuus L.) is one of the main oil plants in the world with an increasingly important ornamental value. It has become one of the primary model plants for genetics research (Knapp et al., 2001). To improve the traits of this plant, radiation or chemicals have been used for mutation induction (Nehnevajova et al., 2007; Skorić et al., 2008), and many mutants have been obtained with new characters, beneficial for breeding new varieties. Commonly, one or two major traits have been altered to upgrade the plant varieties by these means (Ahloowalia et al., 2004).

Alternatively, space flight has been a more effective pathway for inducing a mutation of plants, and up to date, numerous crop plants such as rice, wheat, maize, rape, green pepper, cucumber, tomato, and other plants are undergoing trial planting (Li SZ et al., 2007; Chen et al., 2007; Lu et al., 2008; Visscher et al., 2009). Under these conditions, plant materials were exposed to a vacuum environment with factors of cosmic rays, microgravity, high vacuum, and an interchangeable magnetic field (Kondyurin, 2001; Ruyters and Friedrich, 2006) by using the returned-satellite as a carrier. The morphological traits and genetic background of treated plants could be changed, and after selective seeding and cultivating on earth, new varieties with expected characters could be screened out (Li SZ et al., 2007).

In this experiment, pure sunflower seeds were carried by "Shenzhou IV" and exposed to space conditions in 2002, and then they were grown under natural conditions for mutantscreening. Many plants with new characters were obtained by screening one and one generation from the descendent plants, in which some traits showed stability by successive growth and observation. Whether these characters were due to a variation in the genetic background, or only from the growing conditions, remains to be elucidated. It would be necessary to test their genetic background by molecular markers.

Numerous molecular markers, such as RFLP (Gentzbittel et al., 1994), AFLP (Hongtrakul et al., 1997), and RAPD (Arias and Rieseberg, 1995), have been used to mark plant materials, but simple sequence repeats (SSRs), as a highly reproducible molecular marker, has become one of the most effective markers for population genetic analysis (Knapp et al., 2001; Tang et al., 2003). Thus, SSR markers were used for analyzing the genetic background of the sunflower induced by space flight, and the amplified fragments showing variation in length were sequenced for alignment analysis, in an attempt to provide a scientific basis for breeding new varieties. 


\section{MATERIAL AND METHODS}

\section{Plant materials}

Pure original sunflower seeds were obtained from the Mountain Guangwu, Bazhong City, Sichuan Province, China. They were carried by "Shenzhou IV" for space flight treatment in 2002. Then, seeds were grown in the field individually and visualized. A new variety with new character was inbred, and its descendent plants were grown as a line individually in next generation. Sunflower seeds without treatment were grown under the same conditions, and tested as control. The plant materials investigated in this experiment were descendent plants of the 5th generation grown in 2008 from original treated sunflower seeds.

\section{Analysis of morphological characters of flower and leaf}

In the 6th generation, sunflower plants of interest, including 8 stable mutant lines, were observed and studied. As a special material, the absolute sterile plants were investigated similarly, which could be segregated only from the heterozygous parent plants. The morphological characters of these plants, including floral disc, petal shape, petal color, plant fertility, and leaf shape, were investigated and compared. The typical morphological characters of partial plants were recorded by an Olympus Camera. Plants from seeds free of treatment were used as control.

\section{DNA extraction}

Mature leaves of sunflower sample plants were collected individually for total DNA extraction, according to the report by Li JT et al. (2007). Between 300-400 mg leaf samples were frozen at $-70^{\circ} \mathrm{C}$, then ground into fine powder, after which the materials were extracted in a reagent containing CTAB buffer for $1 \mathrm{~h}$, followed by adding $600 \mu \mathrm{L}$ phenol and chloroform (1:1) into equal volumes of extract solution at $1.5-\mathrm{mL}$ EP tube. After extraction and centrifugation, DNA pellets were precipitated once with pure ethanol, followed by washing twice with $70 \%$ ethanol. Finally, DNA pellets were dissolven in 100 $\mu \mathrm{L}$ TE buffer, and their purity and quality were detected by electrophoresis on $1 \%$ agarose and visualized by the Gel Imaging System. The obtained DNA samples were restored at $-20^{\circ} \mathrm{C}$ for use.

\section{SSR analysis}

A total of thirty-five SSR markers were selected from the linkage map developed by Tang et al. (2002), and were synthesized by Generay Biotech Co., Ltd. (Shanghai, China). The primers were listed as following: ORS545, ORS1112, ORS752, ORS477, ORS924, ORS785, ORS337, ORS309, ORS644, ORS1217, ORS1024, ORS484, ORS1153, ORS840, ORS1043, ORS762, ORS826, ORS1108, ORS1001, ORS759, ORS838, ORS617, ORS541, ORS930, ORS684, ORS818, ORS537, ORS1095, ORS974, ORS386, ORS738, ORS634, ORS821, ORS1241, and ORS988. 
Each PCR system consisted of $1 \mu \mathrm{L} 10-20 \mathrm{ng} / \mu \mathrm{L}$ DNA sample, $2.5 \mu \mathrm{L} 10 \mathrm{X}$ reaction buffer, $1.5 \mu \mathrm{L} 25 \mathrm{mM} \mathrm{MgCl}, 2 \mu \mathrm{L} 0.5 \mathrm{mM}$ dNTPs, $0.5 \mu \mathrm{L}$ forward primer and reverse primer, 1.5 U Taq DNA polymerase (Takara Biotechnology Dalian Co., Ltd., China), and was made up to total a $25-\mu \mathrm{L}$ volume by $\mathrm{ddH}_{2} \mathrm{O}$. PCR was performed on a My Cycler Thermal Cyclera (BIO-RAD) by an initial denaturation of the DNA template at $95^{\circ} \mathrm{C}$ for 4 min, then by 35 cycles touchdown PCR (annealing temperature was increased $0.2^{\circ} \mathrm{C}$ by per cycle) at $95^{\circ} \mathrm{C}$ for $30 \mathrm{~s}, 53^{\circ}-60^{\circ} \mathrm{C}$ for $45 \mathrm{~s}$ and $72^{\circ} \mathrm{C}$ for $50 \mathrm{~s}$, followed by product extension at $72^{\circ} \mathrm{C}$ for $7 \mathrm{~min}$.

PCR products were separated by electrophoresis on 2 or $5 \%$ agarose gel with $0.5 \mathrm{X}$ TBE buffer and 50-bp ladder DNA markers (Takara Biotechnology Dalian Co.). The electrophoretic gel was stained with ethidium bromide and visualized under UV light and then analyzed by Gel doc $2000 \mathrm{~T}_{2} \mathrm{~A}$ (Bio-Rad). The expected fragments of PCR products were harvested and purified from gel using a DNA Purification Kit (Omega), and then ligated into the pMD19-T vector (TaKaRa) at $16^{\circ} \mathrm{C}$ for $2 \mathrm{~h}$. The recombinant molecules were transformed into Escherichia coli competent cells (DH5 $\alpha$ ), and then the recombinant $E$. coli cells were spread on the LB-plate containing $50 \mu \mathrm{g} / \mathrm{mL}$ ampicillin, $200 \mathrm{mg} / \mathrm{mL}$ IPTG and $20 \mathrm{mg} /$ $\mathrm{mL}$ X-gal. Plasmid DNA was isolated and digested by PstI and ScalI to verify the insert size. Plasmid DNA was sequenced by Introvigen Bio-technology Corporation (Shanghai, China). The data of the sequence were uploaded in NCBI (http://www.ncbi.nlm.nih.gov/).

\section{Data analysis}

The sequences were analyzed by the GenScan ${ }^{\circledR}$ software (http://genes.mit.edu/ GENSCAN.html). Homological analysis of the obtained sequences in this experiment, with those in other varieties, was performed with BLAST 2.1 (http://www.ncbi.nlm.nih.gov/blast/). The DNAMAN 6.0 software was used for Multiple Sequence Alignment.

\section{RESULTS}

\section{Variation of morphological characters in flowers and leaves}

In the 6th generation, sunflower plants from 8 stable lines, as well as absolute sterile plants segregated from the heterozygous plants, were observed. Their morphological characters were compared with normal plants, as listed in Table 1. The main characters of the plants were photographed and shown in Figure 1.

By comparing the morphological characters of plants from the 9 samples with normal plants, it was found that their flower color could change from golden to yellow (Figure 1C), light yellow (Figures 1D and H-J), or even to yellowish green (Figure 1B). The ligulate petals of the asexual floret became broadened (Figures 1B and C), or thin (Figures 1F, G, I, and J), while the short tubular petals of amphoteric floret were elongated to some extent (Figures $1 \mathrm{G}$ and J), or they even turned into semi-ligulate petals or ligulate petals, making the phenotype of the whole inflorescence like a chrysanthemum. Similarly, the shape and thickness of leaves varied in some of these plants, i.e., the concavo-convex of leaf aggravated or became thicker (Figures 1L and K). 


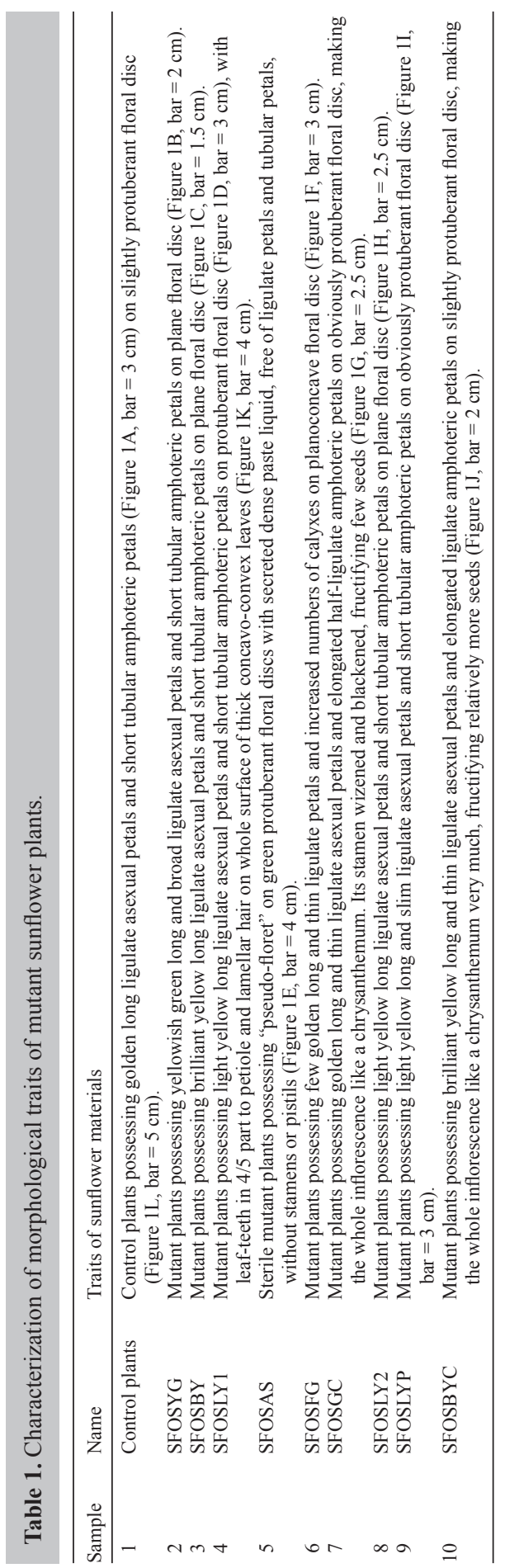




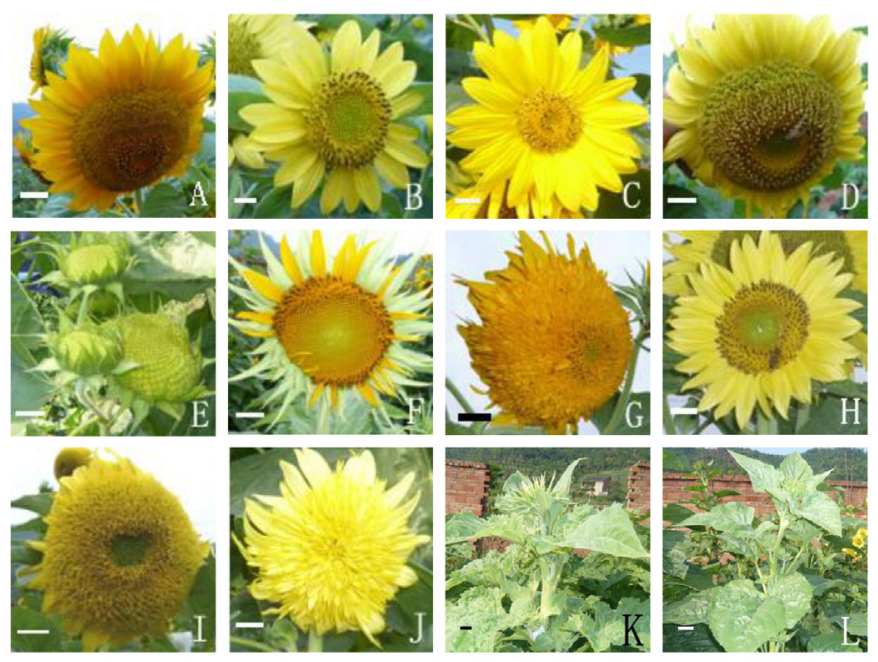

Figure 1. Morphological characters of mutant sunflower line plants treated by space flight. A. and L. Normal plants derived from the seeds free of treatment. B.-K. Mutant plants derived from seeds treated by space flight.

Of interest, we found absolute sterile plants from 3rd to 6th generation, which had neither normal bisexual floret nor unisexual floret, only a "pseudo-floret" could be found on protuberant floral disc (Figure 1E). By dissecting the "pseudo-floret", it was found that pieces of shield-like bracts (intermediate) replaced sepals, petals, anthers, and pistils, subsequently the plant could produce no seeds. Furthermore, the "pseudo-floret" could propagate crazily, i.e., numerous "pseudo-floret" could be proliferated from the position of original "pseudo-floret", making it enlarged gradually (Figures 2A-D). Although the plant with this character showed absolute sterility, new plants with the same character could be found in the next generation, thus it could be deduced that this character was controlled by recessive gene(s), and the character appeared in the next generation probably segregated from heterozygous parent plants.
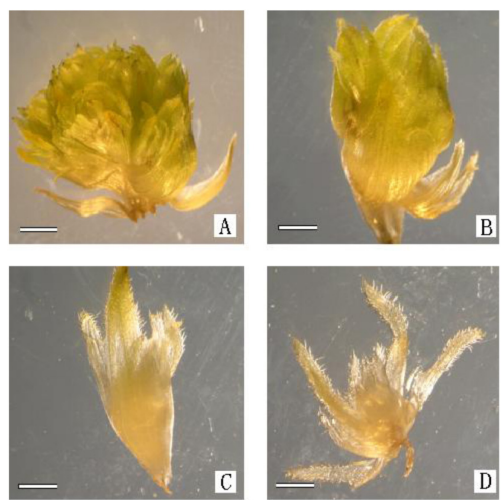

Figure 2. Dissectional observation on "pseudo-floret" on green floral disc of absolute sterile mutant sunflower. A. A whole pseudo-floret from outer part of floral disc of sterile plant, $b a r=2.65 \mathrm{~mm}$. B. A smaller pseudo-floret dissected from part of pseudo-floret shown in A, bar $=2.18 \mathrm{~mm}$. C. Another smaller pseudo-floret dissected from part of pseudo-floret shown in B, bar $=1.07 \mathrm{~mm}$. D. An even smaller pseudo-floret dissected from part of pseudofloret shown in $\mathrm{C}$, bar $=0.38 \mathrm{~mm}$. 


\section{Results analyzed by SSR markers in mutant sunflower plants}

In this experiment, $35 \mathrm{SSR}$ marker primers were used to detect the background of the plants. The results showed that fragments were amplified from all the primers, except ORS752, even with three repeated experiments (data not shown), but most primers detected no variation of fragment in mutant samples, in comparison with normal plants. Variation was only tested from only 4 primers (ORS759, ORS838, ORS840, ORS1112), and the length of the amplified fragments ranged from 180 to $500 \mathrm{bp}$ (Figure 3A-D).
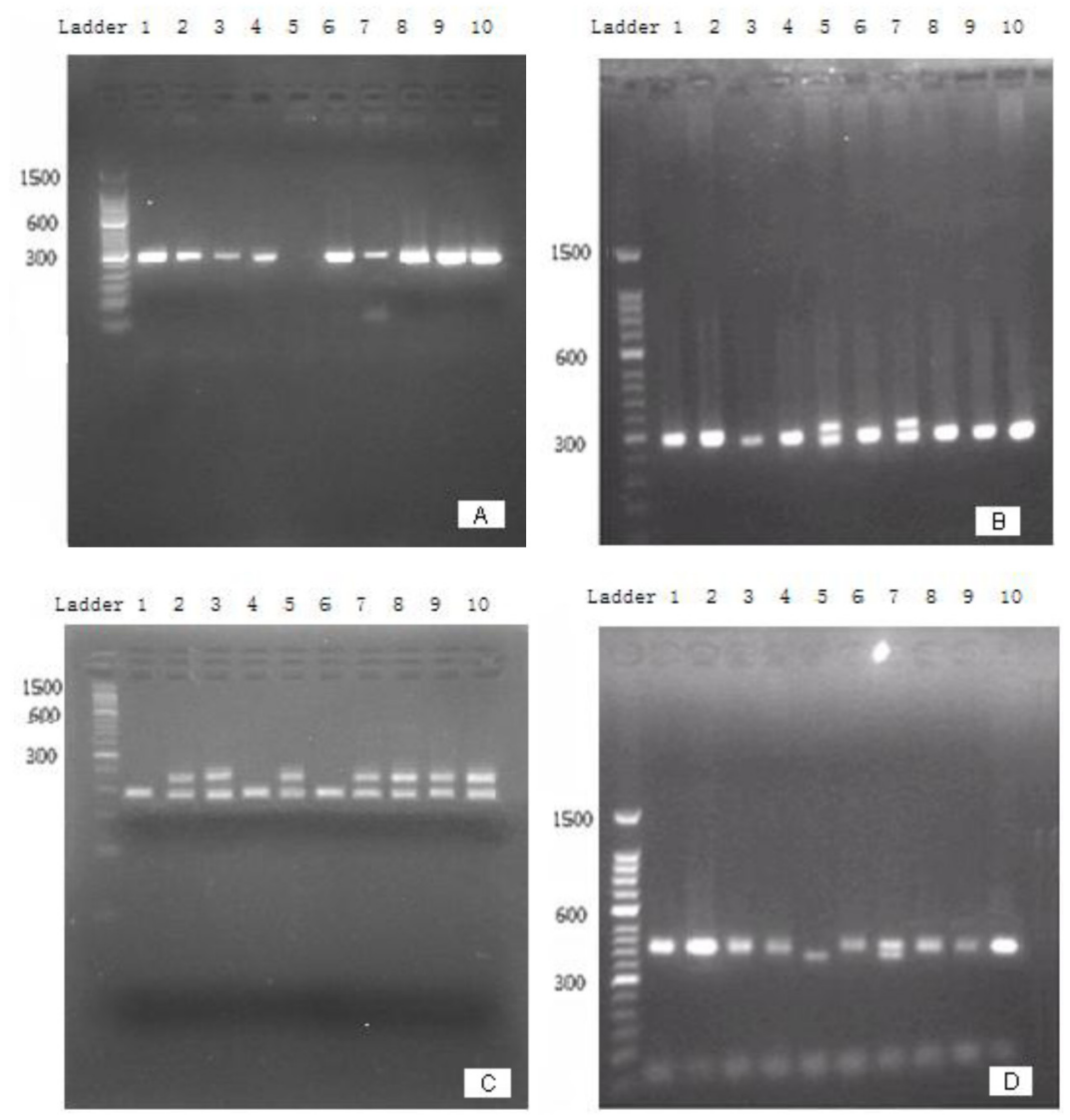

Figure 3. Electrophoretic PCR results from SSR primers on agarose gel. A. Amplified results from primer ORS759 on $5 \%$ agarose gel. B. Results from primer ORS 838 on 2\% agarose gel. C. Results from primer ORS 840 on 5\% agarose gel. D. Results from primer ORS1112 on 2\% agarose gel. Band 1-10 representing amplified bands from lanes 1-10, respectively, corresponding to those in Table 1. Marker is 50-bp ladder (Takara Biotechnology Dalian Co., Ltd.).

Analyzing the amplified products of primer ORS759, it was found that only one coelongate fragment of approximate $300 \mathrm{bp}$ was amplified in 8 mutant samples, similar to that of the control plants in length, but no product was obtained in sample 5 (Figure 2A). Similar length of amplified products was achieved by primer ORS838; however, another fragment of 
about 330 bp was obtained in samples 5 and 7. Concerning primer ORS840, two fragments, at about 180 and $210 \mathrm{bp}$, were amplified in 7 of 9 mutant samples, not different from the control plants. While in samples 4 and 6, only the fragment of about $180 \mathrm{bp}$ was amplified. As regards the primer ORS1112, a similar product of approximately 420 bp was amplified in samples 1-4, sample 6 and samples 8-10, a different fragment of about $380 \mathrm{bp}$ was amplified in sample 5. Of interest, two fragments containing the previous products were obtained in sample 7.

\section{Results of diverse fragment sequencing}

As diverse fragments were detected in the samples by primers ORS759, ORS838, ORS840, and ORS1112, the fragments representing diverse information were extracted again and purified for sequencing and blasting. Approximately $280 \mathrm{bp}$ were amplified by primer ORS759 from all samples, except for sample 5, this fragment amplified in sample 6 was used for sequencing and upload to GenBank ${ }^{\circledR}$ accession No. GQ409933, partially representing the information of the deleted fragment in sample 5.

As one or two fragments were amplified in all the samples by the other three primers, ORS838, ORS840 and ORS1112, these fragments were respectively isolated, sequenced for comparing molecular information. The molecular information from the two fragments amplified in sample 5 by primer ORS 838 indicated that they varied only $6 \mathrm{bp}$ in length, and the short fragment (290 bp, GF101811), might be resulted from the long fragment (296 bp, GF101812) by deleting a T and a TGCTA at the site of $127 \mathrm{bp}$ and the site of 190 to $194 \mathrm{bp}$, respectively.

From the detailed result of ORS840, it could be established that the two fragments had a high homology in sequence information, probably the short fragment (181 bp, GQ409934) was resulted from the long fragment (215 bp, GQ409935) by a deletion of AC(7)TC(10) repeat occurring at 48 to $81 \mathrm{bp}$ and a replacement of $\mathrm{G}$ by $\mathrm{C}$ at the site of $152 \mathrm{bp}$. As regards their homology it was $83.72 \%$ by direct comparison, but reached $99.45 \%$ after ignoring the deleted part of the fragment.

A similar result was obtained in ORS1112, while the deletion in long fragment (382 bp, GQ409936) occurred in two different GA repeat sites. One was AG(2) from 164 to $167 \mathrm{bp}$, and the other was (GA) 16 from 328 to $359 \mathrm{bp}$, accompanied by a replacement of $A$ to $G$ at the site of $322 \mathrm{bp}$, resulting in the short fragment (346 bp, GQ409937). Their homology was as high as $99.71 \%$ after neglecting the deletion, although their original homology was $83.33 \%$.

\section{DISCUSSION}

Since seeds were exposed to space conditions, numerous crop plants such as rice, wheat, maize, and other plants with new characters were screened out in the descendent generation and some new varieties under trial planting (Lu et al., 2008; Visscher et al., 2009). This might be due to the effects of cosmic radiation, zero gravity and other factors in vacuum environment (Cyranoski, 2001). Similarly, after seeds were treated by space flight, various mutant lines of sunflower were obtained by screening one and one generation, providing sources for cultivating new sunflower varieties (Chen et al., 2009). This confirms the high mutagenic efficacy of space flight than irradiation treatment (Wei et al., 2006).

Interestingly, a mutant line with elongated tubular petals was screened in the descendent plants, making the whole inflorescence like a chrysanthemum. Furthermore, this charac- 
ter could have inherited stability, and is significant for cultivating a high quality of ornamental sunflowers. Unfortunately, mutant plants with this character possessed a very low fertility capability, for Helianthus genus is allogamous, with entomophilous fertilization (Frez and Simpson, 1964; Pham-Delegue et al., 1990), thus the over elongated petals could baffle the procedure of entomophilous fertilization, resulting in their low fertility.

Among the mutant plants, another prominent character was absolute sterility, without normal floret, just "pseudo-floret" in capitulum of the plants. It seemed that homologous structure of the "pseudo-floret" to normal floret, such as petals, stamens and pistils all turned into bractea. This mutant appears to be similar to "crazy sepal" mutant in Solarium microdontum (Bamberg, 2006) and sepallata mutant in Arabidopsis (Pelaz et al., 2000), but quite different from previous reports, because it could proliferate dramatically. As a mutant plant with this character had neither normal anthers nor normal pistils, subsequently could not produce descendents. However, new plants with the same character could be found in the next generation from the relative plants, so it could be deduced that this character was controlled by recessive gene(s), because dominant gene(s) should have been lost by only one generation for its absolute sterility, thus the gene(s) will have to be kept heterozygous in mutant plants, and the character appeared in the next generation probably segregated from heterozygous parent plants.

To investigate the genetic variation of the mutants induced by space flight, 35 randomly selected SSR primers were used to test the polymorphism of these plants. The results showed that most primers detected no variation in electrophoretic bands of DNA in these plants. As concerning to the variation detected by the 4 primers, the same length of PCR products was amplified in most of the 10 samples; only one or two samples showed variation in the genetic background, suggesting their homogenous background in general, in consistence with the purity of seeds before exposed to space conditions. To be emphasized, the variation detected by the 4 primers proved the change of genetic background in the plants induced by space flight.

For deeply analyzing the variation of the genetic background, the different PCR products were sequenced for alignment analysis. Until now, only Tang et al. (2002) reported the sequence information amplified by primers ORS759, ORS838, ORS840, ORS1112, and only one fragment was achieved by each of the primers. By comparing the alignment of our results with previous report (Tang et al., 2002), it was found that the homologies of all variation sequences are greater than $92 \%$, or even as high as $99 \%$, indicating the high conservation of genomic sequence of sunflower (Heesacker et al., 2008). Based on the high conservation of the sequences, the analysis of sequence variation showed that deletion, insertion and replacement occurred at some sites in the genomic DNA of sunflower, which was probably the main reason for the major change in morphological characters induced by space flight.

In short, after seeds of sunflower were exposed to space conditions, various mutant plants were screened from the descendent plants. Testing the DNA of the plants by SSR primers indicated the variation of genetic background. The alignment analysis showed that the DNA sequence changed by deletion, insertion and replacement occurred at some sites. Even though the results did not show all principles of the mutation, it did provide partially the ways of DNA transform by space flight, resulting in the changes in morphological characters of sunflower.

\section{ACKNOWLEDGMENTS}

Research supported by the Sichuan Provincial Youth Science and Technology Foun- 
dation (\#2005ZQ026-017), the Sichuan Provincial Key Subject Program (\#SZD0420), and the National Natural Science Fund of China (\#31171587).

\section{REFERENCES}

Ahloowalia BS, Maluszynski M and Nichterlein K (2004). Global impact of mutation-derived varieties. Euphytica 135: 187-204.

Arias DM and Rieseberg LM (1995). Genetic relationships among domesticated and wild sunflowers (Helianthus annuus, Asteraceae). Econ. Bot. 49: 239-248.

Bamberg J (2006). Crazy sepal: A new floral sepallata-like mutant in the wild potato Solanum microdontum Bitter. Am. J. Potato Res. 83: 433-435.

Chen WY, Chen ZY and Yang J (2009). Floral morphological characters and pollination characteristics of sunflower induced by space flight. J. Mianyang Norm. Univ. 28: 56-60.

Chen XD, Lan J and Wang XG (2007). Primary effects on Isatis indigotica after spaceflight. Zhong Yao Cai 30: 381-383. Cyranoski D (2001). Satellite will probe mutating seeds in space. Nature 410: 857.

Frez JB and Simpsom J (1964). The pollination requirements of sunflowers. Emp. J. Exp. Agric. 32: 340-342.

Gentzbittel L, Zhang YX, Vear F, Griveau B, et al. (1994). RFLP studies of genetic relationships among inbred lines of the cultivated sunflower, Helianthus annuus L.: evidence for distinct restorer and maintainer germplasm pools. Theor. Appl. Genet. 89: 419-425.

Heesacker A, Kishore VK, Gao W, Tang S, et al. (2008). SSRs and INDELs mined from the sunflower EST database: abundance, polymorphisms, and cross-taxa utility. Theor. Appl. Genet. 117: 1021-1029.

Hongtrakul V, Huestis GM and Knapp SJ (1997). Amplified fragment length polymorphisms as a tool for DNA fingerprinting sunflower germplasm: genetic diversity among oilseed inbredlines. Theor. Appl. Genet. 95: 400-407.

Knapp SJ, Berry ST and Rieseberg LH (2001). Genetic Mapping Insunflower. In: DNA Markers in Plants (Philips RL and Vasil IK, eds.). Kluwer Academic Publishers, Dordrecht, 379-403.

Kondyurin A (2001). Large-size space laboratory for biological orbit experiments. Adv. Space Res. 28: 665-671.

Li JT, Yang J, Chen DC, Zhang XL, et al. (2007). An optimized mini-preparation method to obtain high-quality genomic DNA from mature leaves of sunflower. Genet. Mol. Res. 6: 1064-1071.

Li SZ, Cao MJ, Rong TZ, Pan GT, et al. (2007). Cytological observation on pollen abortion of genetic male sterile mutant induced by space flight in maize. Fen Zi Xi Bao Sheng Wu Xue Bao 40: 359-364.

Lu WH, Wang XZ, Zheng Q, Guan SH, et al. (2008). Diversity and stability study on rice mutants induced in space environment. Genomics Proteomics Bioinformatics 6: 51-60.

Nehnevajova E, Herzig R, Federer G, Erismann KH, et al. (2007). Chemical mutagenesis - a promising technique to increase metal concentration and extraction in sunflowers. Int. J. Phytoremediation 9: 149-165.

Pelaz S, Ditta GS, Baumann E, Wisman E, et al. (2000). B and C floral organ identity functions require SEPALLATA MADS-box genes. Nature 405: 200-203.

Pham-Delegue MH, Etievant P and Guichard E (1990). Chemicals involved in honeybee-sunflower relationship. J. Chem. Ecol. 16: 3053-3065.

Ruyters G and Friedrich U (2006). Gravitational biology within the German Space Program: goals, achievements, and perspectives. Protoplasma 229: 95-100.

Skorić D, Jocic S, Sakac Z and Lecic N (2008). Genetic possibilities for altering sunflower oil quality to obtain novel oils. Can. J. Physiol. Pharmacol. 86: 215-221.

Tang S, Yu JK, Slabaugh B, Shintani K, et al. (2002). Simple sequence repeat map of the sunflower genome. Theor. Appl. Genet. 105: 1124-1136.

Tang S, Kishore VK and Knapp SJ (2003). PCR-multiplexes for a genome-wide framework of simple sequence repeat marker loci in cultivated sunflower. Theor. Appl. Genet. 107: 6-19.

Visscher AM, Paul AL, Kirst M, Alling AK, et al. (2009). Effects of a spaceflight environment on heritable changes in wheat gene expression. Astrobiology 9: 359-367.

Wei LJ, Xu JL and Wang JM (2006). A comparative study on mutagenic effects of space flight and irradiation of y-rays on rice. Agric. Sci. China 5: 812-819. 